\title{
SUMS OF FOUR SQUARES IN A QUADRATIC RING(1)
}

\author{
BY \\ HARVEY COHN AND GORDON PALL
}

1. Introduction. Let $d$ be a nonsquare integer of the form $4 k+j$ ( $k$ integral, $j=0$ or 1 ). Let $Z$ be the ring of rational integers, $R_{d}$ the ring of numbers $\xi=x_{0}$ $+x_{1} \omega$, where $\omega=(j+\sqrt{d}) / 2, x_{0}$ and $x_{1}$ ranging over $Z$.

In $\S 2$ we will transform the problem of solving

$$
\sigma=\xi_{0}^{2}+\xi_{1}^{2}+\xi_{2}^{2}+\xi_{3}^{2} \quad\left(\xi_{0}, \cdots, \xi_{3} \text { in } R_{d}\right)
$$

where $\sigma=s_{0}+s_{1} \omega$ is a given number in $R_{d}$, into the problem of representing the norm $N \sigma=s_{0}^{2}+j s_{0} s_{1}-k s_{1}^{2}$ by the form

$$
F_{d}=t_{0}^{2}+d t_{1}^{2}+d t_{2}^{2}+d t_{3}^{2} \quad\left(t_{0}, \cdots, t_{3} \text { in } Z\right) ;
$$

with the nontrivial restriction on $t_{0}$ that $b=\left(T \sigma-2 t_{0}\right) / d$ shall be a nonnegative integer, $T \sigma$ denoting the trace $2 s_{0}+j s_{1}$ of $\sigma$. This transformation of the problem uses a recent theorem of Pall and Taussky (see [12]) on the number of representations of a binary quadratic form as a sum of four squares of linear forms.

Relatively little is known of the theory of reduction of quadratic forms over $R_{d}$, as compared with $Z$. As an example, it seems to be true that $d=5$ and 8 are the only positive ring discriminants for which the form $H_{d}=\xi_{0}^{2}+\cdots+\xi_{3}^{2}$ is in a genus of one class; but such results may be hard to prove, by available methods. Hence our transformation of the problem is advantageous. The restriction on the values of $t_{0}$ is no handicap in the study of qualitative questions, concerning the mere representability as a sum of four squares in $R_{d}$. Hence we are able to obtain quite complete results of a qualitatative kind in Part I (cf. Niven [7]). Certain conjectures made by Cohn on the basis of machine calculations (see [1]) are confirmed. We might have simplified some details of proof if we had restricted $R_{d}$ to be maximal, as some writers do. Such restrictions are unnatural: thus it is as significant that a totally positive number $s_{0}+s_{1}(-3)^{1 / 2}$ (with $s_{1}$ necessarily even) is a sum of four squares of numbers $x_{0}+x_{1}(-3)^{1 / 2}$ as the similar fact for $s_{0}+s_{1} \omega, \omega=\frac{1}{2}\left(1+(-3)^{1 / 2}\right)$.

Among the simplest consequences of Part $I$ is that the only values $d$ for which every $\sigma$ satisfying the obviously necessary conditions is a sum of four squares in $R_{d}$ are $d=5,8$, and 12 , and the negative $d$ 's not divisible by 16 . At the referee's suggestion we examined the remaining $d$ 's as regards sums of five or more squares,

Received by the editors September 7, 1961.

(1) Research supported in part by National Science Foundation Grant G-7412. 
and found that if $d<0$ and $16 \mid d$, five squares will suffice; while if $d>12$ there are numbers not sums of any numbers of squares. Of interest is that for positive $d \equiv 1(\bmod 8)$ there exist numbers of arbitrarily large norms, which satisfy the obviously necessary conditions and yet are not sums of four squares in $R_{d}$.

Our treatment is entirely elementary. In Part II we develop an algorithm whereby in certain cases our transformation of the problem permits derivation of formulae for the number of representations of $\sigma$ as a sum of four squares. We carry this through for $d=5,8$, and as far as possible for 12 . These cases had been treated previously, using Hilbert modular functions of two complex variables, by Götzky for $d=5[5 ; 13]$, and by Cohn for $d=8[2]$ and for some cases when $d=12$ $[2 ; 3]$ (making use of Theorem 7, Corollary 2 and (70a) below). Of interest is that, although when $d$ is not a square the weighted number of representations of $n$ by the genus of $F_{d}$ is not a factorable function of $n$, it becomes factorable when $n$ is confined to the multiplicative subgroup of norms $s_{0}^{2}+j s_{0} s_{1}-k s_{1}^{2}$ of discriminant $d$. The algorithm here developed is being applied to forms in two or four variables by one of our students. For the literature in fields, not rings, see [4a].

Notations. Lower case Latin letters denote rational integers; Greek letters elements of $R_{d}$ if not stated otherwise. In Part I, the symbols $d, k, j, \omega, R_{d}, Z$ have the meanings in the introductory paragraph. Throughout, $\sigma=s_{0}+s_{1} \omega$; the norm $N \sigma=s_{0}^{2}+j s_{0} s_{1}-k s_{1}^{2}$; the trace $T \sigma=2 s_{0}+j s_{1} ; r_{4}(n)$ is the number of representations of $n$ as a sum of four squares in $Z_{d} ; r_{4}(\sigma, d)$ the number of representations of $\sigma$ as a sum of four squares in $R_{d}$. In Part I, $O$ and $E$ designate odd or even numbers, which may change from one place to another; $L$ similarly denotes “any" integer of the form $4^{h}(8 n+7), h$ nonnegative, $h$ and $n$ integral.

\section{PART I}

2. The Pall-Taussky formula and its transformation to our problem. For given $s_{0}, s_{1}$ we wish to solve the equation

$$
s_{0}+s_{1} \omega=\left(a_{0}+b_{0} \omega\right)^{2}+\cdots+\left(a_{3}+b_{3} \omega\right)^{2}
$$

in integers $a_{0}, b_{0}, \cdots, a_{3}, b_{3}$. Since $\omega$ is irrational and $\omega^{2}=j \omega+k$, the solution is equivalent to expressing $s_{0}$ in all ways as $a+k b$ in nonnegative integers $a$ and $b$, and finding the integers $a_{i}, b_{i}$ such that

$$
a=\sum a_{i}^{2}, \quad b=\sum b_{i}^{2}, \quad s_{1}-j b=2 \sum a_{i} b_{i} .
$$

Here the summation index $i$ goes from 0 to 3. Now (2) amounts to expressing a binary quadratic form as a sum of four squares of linear forms with integral coefficients:

$$
a x^{2}+\left(s_{1}-j b\right) x y+b y^{2}=\sum\left(a_{i} x+b_{i} y\right)^{2} .
$$

The Pall-Taussky formula is for the number of such expressions of a binary 
quadratic form, and is in our present notations as follows. Necessarily, $s_{1}-j b$ is even. Let $e$ denote the g.c.d. $\left(a,\left(s_{1}-j b\right) / 2, b\right)$, le the g.c.d. $\left(a, s_{1}-j b, b\right)$, so that $l$ is 1 or 2 . Then the Pall-Taussky formula is

$$
\sum_{\left(t_{1}, t_{2}, t_{3}\right)} r_{4}(l h)
$$

where $h=\left(t_{1}, t_{2}, t_{3}, e\right)$, and one term of the sum occurs for each ordered triple $t_{1}, t_{2}, t_{3}$ of integers satisfying

$$
a b-\left(s_{1}-j b\right)^{2} / 4=t_{1}^{2}+t_{2}^{2}+t_{3}^{2} .
$$

Accordingly, $r_{4}(\sigma, d)$ will be obtained from (4) by summing (4) over $b$, or, what is the same thing,

$$
r_{4}(\sigma, d)=\sum_{\left(b, t_{1}, t_{2}, t_{3}\right)} r_{4}(l h)
$$

where $b$ ranges over all nonnegative integers $\left(b>0\right.$ if $s_{0}<0 ; b \equiv s_{1}(\bmod 2)$ if $d$ is odd), and for each such $b$, the $t_{i}$ range over integers satisfying

$$
\left(s_{0}-k b\right) b-\left(s_{1}-j b\right)^{2} / 4=t_{1}^{2}+t_{2}^{2}+t_{3}^{2} .
$$

The product of the left member of $\left(5^{\prime}\right)$ by $d=4 k+j$ simplifies to $N \sigma-t_{0}^{2}$, where $t_{0}=(T \sigma-d b) / 2$. Hence $\left(5^{\prime}\right)$ can be replaced by

$$
N \sigma=t_{0}^{2}+d t_{1}^{2}+d t_{2}^{2}+d t_{3}^{2},
$$

where $t_{0}$ is to be restricted so that $\left(T \sigma-2 t_{0}\right) / d$ is a nonnegative integer $b$. Automatically, $b$ has the parity of $s_{1}$ if $d$ is odd. It is not hard to deduce from (7), written in the form

$$
(T \sigma)^{2}-\left(2 t_{0}\right)^{2}=d\left(s_{1}^{2}+4 t_{1}^{2}+4 t_{2}^{2}+4 t_{3}^{2}\right)
$$

and the fact that $b \geqq 0$, and that $Q_{b} \geqq 0$, where

$$
Q_{b}=\left\{N \sigma-((T \sigma-d b) / 2)^{2}\right\} / d,
$$

that if $d$ is positive, then the solvability of (1) requires that

$$
N \sigma \geqq 0 \text { and } T \sigma \geqq 0 .
$$

In other words, $\sigma$ must be totally positive, a fact otherwise evident. Total positiveness of $\sigma$, and $s_{1}$ even if $d$ is even, are the "trivially necessary" conditions for solvability of (1) when $d$ is positive.

Further, if $d$ is positive and (9) holds, then for any integer $t_{0}$ satisfying (7) and $2 t_{0} \equiv T \sigma(\bmod d)$, the integer $b$ determined by $b=\left(T \sigma-2 t_{0}\right) / d$ is always nonnegative; for, $Q_{b} \geqq 0$ if and only if

$$
\left(T \sigma-2(N \sigma)^{1 / 2}\right) / d \leqq b \leqq\left(T \sigma+2(N \sigma)^{1 / 2}\right) / d,
$$

where, since $(T \sigma)^{2}=4 N \sigma+d s_{1}^{2}$, the left member is nonnegative. Thus when $d$ is 
positive, $b$ is confined to a finite interval of length $4(N \sigma)^{1 / 2} / d$. But if $d<0$, $Q_{b} \geqq 0$ if and only if $b \geqq\left(T \sigma-2(N \sigma)^{1 / 2}\right) / d$, an interval of infinite length. This advantage to $d$ negative is somewhat compensated by the fact that, as ( $\left.7^{\prime}\right)$ shows, changing $t_{0}$ to $-t_{0}$ then changes the sign of $b$.

Our basic result will be used so often that we state it as a theorem:

THEOREM 1. Let $\sigma$ have $s_{1}$ even if $d$ is even. Then (1) is solvable if and only if there exists a nonnegative integer $b$ for which $Q_{b}$ is a nonnegative integer not of the form $L$.

The condition $2 t_{0} \equiv T \sigma(\bmod d)$ is satisfied for all integral solutions $t_{0}, \cdots, t_{3}$ of (7) in certain cases, and for exactly half the solutions in certain others:

THEOREM 2. If $d$ or $\frac{1}{4} d$ is a prime, then if (9) holds, and $s_{1}$ is even when $d$ is even, (1) is solvable if and only if $N \sigma$ is represented by $F_{d}$.

Proof. For any solution of (7),

$$
(T \sigma)^{2} \equiv\left(2 t_{0}\right)^{2}(\bmod d) \text { if } d \text { is odd, } \quad s_{0}^{2} \equiv t_{0}^{2}(\bmod d) \text { if } d \text { is even. }
$$

Hence if $d$ is an odd prime, $2 t_{0} \equiv T \sigma(\bmod d)$ holds for both signs of $t_{0}$ if $d \mid N \sigma$, by choice of that sign otherwise. If $d=4 p\left(p\right.$ prime), we need $t_{0} \equiv s_{0}(\bmod 2 p)$; this holds automatically if $p \mid s_{0}$, by choice of sign of $t_{0}$ otherwise.

3. The universal discriminants. A discriminant $d$ is called universal if (1) is solvable for every $\sigma$ satisfying the obviously necessary conditions, (9) if $d>0$, $2 \mid s_{1}$ if $2 \mid d$. For field discriminants, some restrictions on $d$ were known quite early but there probably is no exact reference prior to 1928 [5].

LeMma $1 . d$ is not universal if $d$ is divisible by 16 or $d>12$.

Proof. It will be convenient, when $d=4 k$, to set

$$
\begin{aligned}
& k=2^{u} k^{\prime}, k^{\prime} \quad \text { odd, } u \geqq 0 ; \quad N \sigma=2^{w} s, \quad s \text { odd, } w \geqq 0 ; \\
& s_{0}=2^{v} s_{2}, s_{2} \quad \text { odd, } v \geqq 0 ; \quad s_{1}=2^{g+1} s_{3}, \quad s_{3} \text { odd, } g \geqq 0 ;
\end{aligned}
$$

the last two if $s_{0} s_{1} \neq 0$, otherwise we can take $v$ or $g$ arbitrarily large.

To obtain a number $\sigma$ for which (1) is unsolvable, when $16 \mid d$, we can take $g=0$, and $v=\frac{1}{2}(u+2)(>1)$ or $v=\frac{1}{2}(u+3)(>2)$ according as $u$ is even or odd. Then $t_{0} / 2^{v}=s_{2}-2^{u+1-v} k^{\prime} b$ is odd for every integer $b$, and $N \sigma=2^{2 v} s_{2}^{2}-d s_{3}^{2}$. Hence $N \sigma-t_{0}^{2}$ has the excluded form $d L$ for every integer $b$.

If $d$ is positive take $\sigma=s_{0}+(2-j) \omega$, where $s_{0}$ is the positive integer determined by the inequalities

$$
\left(2 s_{0}-1\right)^{2}<d<\left(2 s_{0}+1\right)^{2}(d \text { odd }), \quad\left(s_{0}-1\right)^{2}<4 k<s_{0}^{2}(d \text { even }) .
$$

Then $2 s_{0}+1<2+d^{1 / 2}, s_{0}<1+(4 k)^{1 / 2}$ respectively, and hence (cf. (10)) 
$\left(T \sigma+2(N \sigma)^{1 / 2}\right) / d<\left(2+d^{1 / 2}+\left(4+4 d^{1 / 2}\right)^{1 / 2}\right) / d$ or $\left(2+4 k^{1 / 2}+2\left(1+4 k^{1 / 2}\right)^{1 / 2}\right) / d<1$, provided $d>12$. Hence (1) is unsolvable for this $\sigma$ if $d>12$.

Lemma 2. If $d>12$, the number $s_{0}+(2-j) \omega$ defined above is not a sum of five, or indeed any number of, squares of elements of $R_{d}$.

Proof. We will suppose $j=1$, the proof for $j=0$, starting with the inequality $0<s_{0}-2 k^{1 / 2}<1$, being similar. We have

$$
0<s_{0}+\bar{\omega}<1, \text { where } \bar{\omega}=\left(1-d^{1 / 2}\right) / 2 \text {. }
$$

Hence if $s_{0}+\bar{\omega}$ is a sum of squares $\sum \alpha^{2}$, all the terms satisfy $0 \leqq \alpha^{2}<1$, and hence for any nonzero term $\alpha^{2}, \bar{\alpha}^{2}>1$. Since $s_{0}+\bar{\omega}$ is not a sum of four squares, at least five of the terms $\alpha^{2}$ are not zero, and being less than 1 each of these terms is irrational. If one of these, say $\alpha^{2}$, is $(t+u \sqrt{d}) / 2$, then $\bar{\alpha}^{2}=$ $(t-u \sqrt{d}) / 2$, and $\bar{\alpha}^{2}-\alpha^{2}=-u \sqrt{d} \geqq \sqrt{d}$ (since $\left.\bar{\alpha}^{2}>\alpha^{2}\right)$. Subtracting from $s_{0}+\omega=\sum \bar{\alpha}^{2}$, we have $\sqrt{d} \geqq 5 \sqrt{d}$, a contradiction.

THEOREM 3. $d$ is universal if and only if $d$ is 5,8 , or 12 , or negative but not divisible by 16. If $d>12$ there exists a totally positive number $s_{0}+(2-j) \omega$ which is not a sum of squares; if $d<0$ and $16 \mid d$, every number (with $s_{1}$ even) is a sum of five squares.

Since as noted after (10) the interval for $b$ is infinite, the proof for $d<0$ is an immediate deduction from Theorems $4,5,6$, along with observation (for the last part of Theorem 3) that either $\sigma$ or $\sigma-1$ will not satisfy (13). We assume $d>0$. By Lemma 1, $d$ can only be 5, 8, or 12 , and by Theorem 2 it suffices to show that $F_{d}$ represents all positive norms $s_{0}^{2}+j s_{0} s_{1}-k s_{1}^{2}\left(s_{1}\right.$ even if $d=8$ or 12$)$.

If $d=5$ no norm is double an odd. It therefore suffices to treat odd norms of the forms $5 q+0,4$, or 16 , with $q=8 k+7$; note $5 q-25, q-5 \neq L ; 5 q+4-9$, $q-1 \neq L ; 5 q+16-1, q+3 \neq L$. If $d=8$, note first that $F_{2}$ represents all positive integers: thus, $2 n+0,1, n=4^{h}(8 k+7) ; 2 n-4, n-2 \neq L ; 2 n+1-9$, $n-4 \neq L$ if $h=0,1 ; 2 n+1-25, n-12 \neq L$ if $h \geqq 2$. Hence $F_{8}$ represents all even norms. There remain only odd norms $8 n+1, n=4^{h}(8 k+7) ; 8 n+1-9$, $n-1 \neq L$ if $h=0,1 ; 8 n+1-25, n-3 \neq L$ if $h \geqq 2$. Let $d=12$. First, $F_{12}$ represents all even norms, since $F_{3}$ represents all positive $3 n+0,1$; for if $n=$ $4^{h}(8 k+7)$, use $3 n-9, n-3 \neq L$ if $h \geqq 1 ; 3 n-36, n-12 \neq L$ if $h=0 ; 3 n+1-4$, $n-1 \neq L$ if $h=0,1 ; 3 n+1-16, n-5 \neq L$ if $h \geqq 2$. For odd norms $12 n+1$, if $n=4^{h}(8 k+7), 12 n+1-25, n-2 \neq L$.

Previous proofs of universality for $d=5$ and 8 used modular functions; for $d=12$, there is a previous proof $[2 ; 3]$ only if $\sigma$ is even or $N \sigma$ large.

4. Further analysis of Theorem 1. Assume $\sigma$ to satisfy the necessary conditions (9), and $s_{1}$ even if $d$ is even. 
If $d>0$ the interval (10) contains at least $q$ integers $b$ (with the parity of $s_{1}$ if $d$ is odd) if

$$
\left.N \sigma \geqq q^{2} d^{2} / 4 \text { ( } d \text { odd }\right), \quad N \sigma \geqq q^{2} d^{2} / 16 \text { ( } d \text { even). }
$$

If it can be shown that $Q_{b} \neq L$ for at least one of every $q$ (or $2 q$, if $d$ is odd) consecutive integers $b$, then it will follow that (1) is solvable if $d<0$, and when (12) holds if $d>0$.

Theorem 4 will give the cases in which $Q_{b}$ has the form $L$ for every integer $b$, so that (1) is surely not solvable. In all other cases Theorems 5 and 6 will prove the existence of an integer $q$ (which we have tried to make best possible in each case) with the aforestated property. Hence in these other cases (1) is solvable if $d<0$, or (12) holds and $d>0$.

\section{4a. Cases in which $Q_{b}=L$ for every $b$.}

THEOREM 4. Let $d, s_{1}$ be even. Then $Q_{b}$ has the excluded form $L$ for every integer $b$ in the following cases:

$$
w<2 v, w<2 u ; w>2 v, u \geqq v ; w=2 v, u-v>y ;
$$

which occur only if $32|d, 16| d, 128 \mid d$ respectively. Here we use the notations in (11), and also if $w=2 v, s-s_{2}^{2}=k^{\prime} 2^{y} m, m$ odd, $y \geqq 0$, with the convention when $s_{0}, s_{1}$ or $s-s_{2}^{2}$ vanishes, that $v$, $g$, or $y$ is "large."

Proof. We can write

$$
2^{w} s=2^{2 v} s_{2}^{2}-2^{u+2+2 g} s_{3}^{2} k^{\prime}, \quad c=2^{v} s_{2}-2^{u+1} k^{\prime} b,
$$

and have that $Q_{b}$ is not of the form $L$ if and only if

$$
2^{w_{s}}-c^{2} \text { is not of the form } 2^{u} k^{\prime} L \text {. }
$$

CASE (13 $) . w=u+2+2 g$ by (14), $s=2^{2 v-w} s_{2}^{2}-k^{\prime} s_{3}^{2}$. Hence if $2 v-w \geqq 3$, $s \equiv-k^{\prime} \bmod 8$ and $2^{w+3} \mid c^{2}$ for every $b$. If $2 v-w=1$ or $2, s \equiv 2^{2 v-w}-k^{\prime} \bmod 8$, $2^{-w} c^{2} \equiv 2^{2 v-w}(\bmod 8)$ for all $b$. CASE $\left(13_{2}\right)$. By $(14), u+2+2 g=2 v, u$ is even, $c=2^{v} c^{\prime}$ with $c^{\prime}=s_{2}-2^{u+1-v} k^{\prime} b$ odd $2^{w} s=2^{2 v}\left(s_{2}^{2}-k^{\prime} s_{3}^{2}\right), 2^{w-2 v} s-c^{\prime 2} \equiv$ $-k^{\prime}(\bmod 8) . \operatorname{CASE}\left(13_{3}\right) \cdot w=2 v<u+2+2 g, s-s_{2}^{2}=-2^{u+2+2 g-2 v} k^{\prime} s_{3}^{2}$. Hence $y \equiv u \bmod 2, m \equiv 7 \bmod 8$. Thus (15) is again demonstrated, since $u+2-v$ $\geqq y+3$ and

$$
s-2^{-2 v} c^{2}=k^{\prime} 2^{y}\left\{m+2^{u+2-v-y} s_{2} b-2^{2 u+2-2 v-y} k^{\prime} b^{2}\right\} .
$$

If $\left(13_{1}\right)$ holds, $2 u-1 \geqq u+2, u \geqq 3$. If $\left(13_{2}\right)$ holds, $2 u \geqq 2 v \geqq u+2, u \geqq 2$. If (133) holds, $u-v>y \geqq 1$ and $y=u+2+2 g-2 v$; hence $u \geqq 5$.

\section{4b. Sufficient conditions for solvability of (1) when $d$ is odd.}

THEOREM 5. Let $d$ be odd. Then (1) is solvable when $\left(12_{1}\right)$ holds, with 


$$
\begin{aligned}
& q=2, \quad \text { if } N \sigma \text { is odd; or if } N \sigma \equiv 0, d \equiv 5(\bmod 8) \\
& q=4, \quad \text { if } N \sigma \equiv 4, d \equiv 5(\bmod 8) \\
& q=2^{[w / 2]}, \text { if } N \sigma=2^{w} s, s \text { odd }, w>0, d \equiv 1(\bmod 8) .
\end{aligned}
$$

Proof. The numbers $B=(T \sigma-d b) / 2$ form an arithmetic progression with odd common difference $d$, so that each residue class $\bmod 2^{n}$ occurs once in $2^{n}$ consecutive terms. We must assure the existence of a $B$ for which $N \sigma-B^{2}$ is not of the form $d L$.

(i) If $N \sigma$ is odd we have $q=2$. For if $N \sigma \equiv 3, N \sigma-O^{2} \equiv 2$; if $N \sigma \equiv 1$, $N \sigma-E^{2} \equiv 1(\bmod 4) .(O, E$ designate odd and even numbers respectively.)

(ii) If $d \equiv 1(\bmod 8)$ and $N \sigma=2^{w} s\left(w, s\right.$ odd), we can take $q=2^{(w-1) / 2}$, and in general not less. For,

$$
2^{w} s-\left(2^{(w-1) / 2} O\right)^{2}=2^{w-1}(4 n+1), 2^{w} s-\left(2^{(w-1) / 2} E\right)^{2}=2^{w} O .
$$

But if $0 \leqq c<(w-1) / 2,2^{w} s-\left(2^{c} O\right)^{2}=2^{2 c}(8 n-1)$ has the excluded form $d L$.

(iii) If $d \equiv 1(\bmod 8)$ and $N \sigma=2^{2 f+2} s$ ( $s$ odd, $\left.f \geqq 0\right)$, we can take $q=2^{f+1}$. For, $N \sigma-\left(2^{f} O\right)^{2}=2^{2 f}(8 n+3)$.

(iv) If $d \equiv 5(\bmod 8)$ it is elementary that 2 cannot divide $N \sigma$ to an odd exponent. If $N \sigma \equiv 4(\bmod 8), N \sigma-O^{2}$ has the excluded form $d L$. But one of $4(4 n+1)$ $-(2 E)^{2}=4(4 n+1), 4(4 n+3)-(2 O)^{2}=4(4 n+2)$ applies; hence $q=4$.

(v) If $d \equiv 5(\bmod 8)$ and $8 \mid N \sigma, N \sigma-O^{2}=8 n+7 \neq d L$; hence $q=2$.

4c. Note on discriminants of the form $8 n+1$.

THEOREM 5'. If $d$ is positive and $\equiv 1(\bmod 8)$ there exist totally positive $\sigma$ of arbitrarily large norms for which (1) is not solvable.

This is suggested by (17) since $q$ increases with the power of 2 in $N \sigma$, but of course this is no proof. To prove the theorem notice first that if $t_{0}, \cdots, t_{3}$ are integers for which $F_{d}$ is divisible by 8 , then $t_{0}, \cdots, t_{3}$ are even. Hence if $F_{d}$ fails to represent one even norm $n$, it does not represent the infinitely many $4^{k} n$. It remains then only to prove the following lemma.

Lemma. If $d \geqq 17, d \equiv 1(\bmod 8)$, then there exists between 1 and $d$ an even nonsquare integer of the form $x^{2}+x+(1-d) / 4$.

Proof. The condition $0<x^{2}+x+(1-d) / 4<d$ is satisfied if

$$
\left(-1+d^{1 / 2}\right) / 2<x<\left(-1+(5 d)^{1 / 2}\right) / 2,
$$

the even squares between 0 and $d$ are $(2 z)^{2}$ where $1 \leqq z<d^{1 / 2} / 2$; and

$$
\left((5 d)^{1 / 2}-d^{1 / 2}\right) / 2-1>d^{1 / 2} / 2 \text { if } d \geqq 41 \text {. }
$$

We can use $2^{2}+2-4$ for $d=17$, and $4^{2}+4-8$ for $d=33$.

We will examine $d=17$ in detail. By Theorem 2 we have only to study the norms 
represented by $F_{17}$. By the foregoing we can confine our first attention to norms not divisible by 8 ; and by Theorem $5, q=1$ or 2 for such norms, so that we can assume $N \sigma \leqq 289$. A norm $s_{0}^{2}+s_{0} s_{1}-4 s_{1}^{2}$ is an integer of the form $17 n+x^{2}$ $(x=0,1, \cdots, 8)$, and cannot be divisible to an odd exponent by any prime $p$ such that $(17 \mid p)=-1$; the last applies in particular to $p=3,5$, and 7 . In $F_{17}$ we can take $t_{0}=x$ provided $n$ is represented by $t_{1}^{2}+t_{2}^{2}+t_{3}^{2}$. We have therefore left to examine $n=-1$ with $x=5,6,7,8 ; n=-2$ with $x=6,7,8 ; n=-3$ with $x=8$; and $n=7$ and 15 . It will be found that all the numbers $17 \cdot 7+x^{2}$ and $17 \cdot 15+x^{2}$ are divisible to an odd exponent by 3,5 , or 7 , except for 128 , not represented by $F_{17} ; 144$ and 256 , obviously represented; $200=8^{2}+17 \cdot 8,263=7^{2}+17 \cdot 11$, $288=4^{2}+17 \cdot 16,271=13^{2}+17 \cdot 6$. Two numbers 15 and 30 , obtained for $n=-2$ or -3 , are also eliminated. We have thus left to consider only the numbers

$$
2^{2 z+1}, 13,19 \text {, and } 47 \text {, }
$$

it being noted that 52,76 , and 188 are represented by $F_{17}$. The last two statements of the following theorem can be verified without difficulty.

THEOREM 5". If $d=17$, the only totally positive numbers $\sigma$ for which (1) is not solvable are those whose norms are listed in (18). Those of norms $2^{2 z+1}(z \geqq 3)$ can be expressed as a sum of five squares in $R_{17}$. Those of norms $2,8,32,13,19$, and 47 cannot be expressed as the sum of any number of squares.

$4 d$. Sufficient conditions for solvability of (1) when $d$ is even. The cases remaining from Theorem 4 can be formulated as $w \geqq 2 u, u<v, w \neq 2 v$; and $w=2 v, u-v \leqq y$. Hence they can be reformulated thus: $w \geqq 2 u, u<v$; and $w=2 v, 0 \leqq u-v \leqq y$. It will be noted in the following theorem that $q$ is unbounded with the power of 2 in $N \sigma$ when the odd part of $d$ has the form $8 n+1$. In all other cases there is a constant $c$ such that (cf.(12)) (1) is solvable if $N \sigma$ $\geqq c d^{2}, \sigma$ being totally positive and $s_{1}$ even. The largest such $c$, given by $q=5$, is $25 / 16$. The notations of Theorem 4 are still used in Theorem 6.

THEOREM 6. Assume d even, $s_{1}$ even. We can take $q$ as follows:

$1^{\circ}$. If $w=2 u+2 f(f \geqq 0)$ and $u<v$, then $q=2$ except that

$q=2^{f-2}$ if $u$ is even, $k^{\prime} \equiv 1(\bmod 8), f>2 ; q=4$ if $f=1, u$ even,

$s \equiv-k^{\prime} \equiv 1(\bmod 4)$, or if $f=2, u$ even, $k^{\prime} \equiv 5(\bmod 8)$.

$2^{\circ}$. If $w=2 u+1+2 f(f \geqq 0)$ and $u<v$, then $q=2$ except that

$q=2^{f-1}$ if $k^{\prime} \equiv 1(\bmod 8), u$ even, $f \geqq 1$;

$q=1$ if $k^{\prime} \equiv 1+2$ s or $5(\bmod 8), u$ even, $f \geqq 1$.

$3^{\circ}$. If $0 \leqq u-v \leqq y, w=2 v, s-s_{2}^{2}=k^{\prime} 2^{y} m$ (hence $m \equiv 7 \bmod 8, y-u$ even $)$, then

$q=2$ if $u-v=y$; or $u-v<y-1, v$ odd, $u>v$; or $u-v=y-1, y>1$;

$q=3$ if $u=v$ and $y=1$; or $u-v<y-1$ and $v$ is even;

$q=5$ if $v$ is odd, $u=v$ and $y>1$. 
Proof. In $1^{\circ}$ and $2^{\circ}, c=2^{u+1} c^{\prime}$, where $c^{\prime}=2^{v-u-1} s-k^{\prime} b$ runs over an arithmetic progression with odd common difference $k^{\prime}$. We are to choose $b$ so that

$$
2^{w} s-2^{2 u+2} c^{\prime 2} \neq 2^{u} k^{\prime} L .
$$

If $w=2 u$ or $2 u+1$ this becomes

$$
s-2^{2 u+2-w} c^{\prime 2} \neq 2^{u-w} k^{\prime} L .
$$

Since $w<2 v, w=u+2+2 g$, so that $u-w$ is even. Also,

$$
s=-k^{\prime} s_{3}^{2}+2^{2 v-w} s_{2}^{2} \equiv-k^{\prime} \text { or }-k^{\prime}+2^{2 v-w}(\bmod 8) \text {. }
$$

Hence (19) holds only by choice of parity of $c^{\prime}$, whence $q=2$.

If $w=2 u+2 f, f \geqq 1$, we must secure

$$
2^{2 f-2} s-c^{\prime 2} \neq 2^{-u} k^{\prime} L
$$

Consider $f=1$. One of $c^{\prime}$ odd or even will serve (hence $q=2$ ) if $u$ is odd; or $u$ is even, $s \equiv 3(\bmod 4)$; or $u$ is even, $s \equiv k^{\prime} \equiv 1(\bmod 4)$. But if $u$ is even and $s \equiv-k^{\prime} \equiv 1(\bmod 4)$, we take $q=4$, using $s-(2 n)^{2}(n$ odd or even).

Consider $f>1$. We can use $c^{\prime}=2^{f-2} O$ if $u$ is odd or $k^{\prime} \not \equiv 5(\bmod 8) ; 2^{f-1} O$ if $u$ is even and $s \equiv 3(\bmod 4) ; 2^{f-1} E$ if $u$ is odd or $s \equiv k^{\prime} \equiv 1(\bmod 4) ; 2^{r} O$ $(0 \leqq r \leqq f-3)$ if $u$ is odd or $k^{\prime} \not \equiv 1(\bmod 8)$. Hence $q=2$ if $u$ is odd; or $f=2$, $k^{\prime} \not \equiv 5(\bmod 8) ;$ or $k^{\prime} \equiv 3(\bmod 4) ;$ or $u$ even, $k^{\prime} \equiv 5(\bmod 8), f>2$. But $q=2^{f-2}$ if $u$ is even, $k^{\prime} \equiv 1(\bmod 8), f>2 ; q=4$ if $u$ is even, $k^{\prime} \equiv 5(\bmod 8), f=2$.

If $w=2 u+2 f+1(f \geqq 1)$, we must secure $2^{2 f-1} s-c^{\prime 2} \neq 2^{-u} k^{\prime} L$. We can use $c^{\prime}=2^{f-1} O$ if $u$ is odd or $k^{\prime} \neq 2 s-1(\bmod 8) ; c^{\prime}=2^{f-1} E$ if $u$ is even; $c^{\prime}=2^{r} O$ $(0 \leqq r \leqq f-2)$ if $k^{\prime} \not \equiv 1(\bmod 8)$ or $u$ is odd. Hence $q=1$ if $u$ is even and $k^{\prime} \equiv$ $1+2 s$ or $5(\bmod 8) ; q=2$ if $u$ is odd or $k^{\prime} \equiv 1-2 s(\bmod 8) ; q=2^{f-1}$ if $k^{\prime} \equiv 1$ $(\bmod 8), u$ even.

Consider $w=2 v, u \geqq v$. Then $w<u+2+2 g$ and $y=u+2+2 g-2 v$, $m \equiv 7(\bmod 8)$. We can use (16), with now $u-v \leqq y$. If $u-v=y, b$ odd will serve, hence $q=2$. If $u-v=y-1,7+2 b-2^{y} k^{\prime} b^{2}$ is not of the form $L$ if $b$ is odd, unless $y=1$; and in the last case, $b \equiv 2$ and one of $b \equiv 1,3(\bmod 4)$ will do, so that $q=3$. If $u-v<y-1$, then for all values of $b, 2^{u+2-v} \mid s-c_{b}^{2}$, where $c_{b}=s_{2}-2^{u+1-v} k^{\prime} b$. We can suppose then that for a certain $b, Q_{b}$ is of the form $L$, and hence $s-c_{b}^{2}=k^{\prime} 2^{z}(8 n+7), z \equiv u(\bmod 2), u+2-v \leqq z$. Then, trying $b+1$, we consider

$$
s-\left(c_{b}-2^{u+1-v} k^{\prime}\right)^{2}=k^{\prime} 2^{u-v+2}\left\{2^{z-u+v-2}(8 n+7)+c_{b}-2^{u-v} k^{\prime}\right\} .
$$

Thus $Q_{b+1}$ is not of the form $L$ if $v$ is odd and $u>v$; hence $q=2$. If $v$ is even, $q=3$, since $s-\left(c_{b}-2^{u+1-v} 2 k^{\prime}\right)^{2}$ is not of the excluded form, being equal to

$$
\begin{array}{ll}
k^{\prime} 2^{u-v+3}\left\{2^{z-u+v-3}(8 n+7)+c_{b}-2^{u-v+1} k^{\prime}\right\} & \text { if } z>u-v+2, \\
k^{\prime} 2^{u-v+2}\left\{8 n+7+2 c_{b}-2^{u-v+2} k^{\prime}\right\} & \text { if } z=u-v+2 .
\end{array}
$$


Finally, if $v$ is odd and $u=v, q=5$ is obtainable, as we can use

$$
\begin{aligned}
s-\left(c_{b}-2^{u-v+1} 4 k^{\prime}\right)^{2} & =8 k^{\prime}\left(8 n+7+2 c_{b}-8 k^{\prime}\right) & & \text { if } z=3, \\
& =16 k^{\prime}\left(2^{z-4}(8 n+7)+c_{b}-4 k^{\prime}\right) & & \text { if } z>4 .
\end{aligned}
$$

4e. Lists for some discriminants, of all totally positive numbers $\tau$, with $s_{1}$ even if $d$ is even, for which (1) is not solvable. These lists form a small part of the unpublished output of [1]; the proof that the lists are complete is easily given using the foregoing theorems. By Theorem 2 it suffices to list the norms when $d$ or $\frac{1}{4} d$ is a prime. In other cases, noting that $\sigma$ and $\sigma \theta^{2}$ behave alike ( $\theta$ denoting a unit in $R_{d}$ ), we list one $\sigma$ from each such equivalence class. Notice the example, when $d=24$, of two numbers $(1$ and $5+2 \omega)$ of the same norm, one of which is, the other not, a sum of four squares. In view of the theorem $[6 ; 7]$ that if $\sigma$ is not a sum of five or fewer squares, then $\sigma$ cannot be expressed as a sum of any number of squares, it is interesting to give the cases in the following lists in which $\sigma$ is a sum of five squares: norm 92 when $d=13$; norms 281 and 284 when $d=40$; norms 308 (both $22+4 \omega$ and $88+26 \omega$ ) and 317 when $d=44$. The largest observed ratio $N \sigma: d^{2}$ for which $\sigma$ was not a sum of four squares was for $\sigma=74+26 \omega$ with $d=29$, where $N \sigma / d^{2}$ was $2668 / 841$.

$d=13:$ norms $3,12,23,92$. $d=24: 5+2 \omega$ of norm $1,6+2 \omega$ of norm 12 .

$d=28$ : norms 8 and 21. $d=44$ : norms $5,20,37,56,77,308,317$.

$d=40: 7+2 \omega, 8+2 \omega, 9+2 \omega, 10+2 \omega, 18+2 \omega, 21+4 \omega$, of respective norms $9,24,41,60,284$, and 281 .

\section{PART II}

Formulae for the number of solutions of (1) when $d=5,8,12$.

5. Formulae for the number of representations of $n=N \sigma$ by $F_{d}$. Since the discriminant of $F_{d}$ is not a square, the weighted number of representations of a positive integer $n$ by the genus of $F_{d}$ is not a factorable function of $n$. It is interesting then that this weighted number of representations becomes factorable, in the cases $d=5,8,12$ (and perhaps more generally), if $n$ is restricted to the set of numbers represented by the principal binary form of discriminant $d$. (These numbers, of course, from a multiplicative semigroup.)

If $d=5, N \sigma$ can be given the notation

$$
n=s_{0}^{2}+s_{0} s_{1}-s_{1}^{2}=2^{2 u} 5^{v} m ; u, v \geqq 0 ; m \equiv 1 \text { or } 9(\bmod 10) .
$$

A classical formula [4] for the number of representations of $2^{a} 5^{b} m$ by $F_{5}$ is $k_{2} k_{5} k_{m}$, where

$$
k_{2}=\left(2^{a+1}-(-1)^{a} 5\right) / 3, \quad k_{5}=5^{b}+(-1)^{a}(m \mid 5), \quad k_{m}=\sum_{m=q q^{\prime}}\left(q^{\prime} \mid 5\right) q .
$$

Notice that $k_{5}$ depends on the factors other than 5 in $n$, so that the formula is not factorable. But if $n$ is a norm (as in (20)), the formula reduces to $2 g\left(2^{2 u} 5^{v} m\right.$ ), 
where $g(n)$ is the factorable function defined for every positive integer $n$ as follows:

$$
\begin{aligned}
g(1) & =1, g\left(n_{1} n_{2}\right)=g\left(n_{1}\right) g\left(n_{2}\right) \text { if }\left(n_{1}, n_{2}\right)=1 ; \\
g\left(2^{2 u}\right) & =\left|2^{2 u+1}-5\right| / 3, g\left(2^{2 u+1}\right)=0, g\left(5^{v}\right)=\left(5^{v}+1\right) / 2, \\
g(m) & =\sum_{m=q q^{\prime}}\left(q^{\prime} \mid 5\right) q .
\end{aligned}
$$

If $d=8, N \sigma$ can be given the notation

$$
n=s_{0}^{2}-8 s_{3}^{2}=2^{u} m \text {, where } u=0, m \equiv 1(\bmod 8) \text {, or } u \geqq 2, m \equiv \pm 1(\bmod 8) \text {. }
$$

The formula [4] for the number of representations of $n$ by $F_{8}$ reduces when $n$ is a norm to $2 g(n)$, where $g(n)$ can be defined for all positive integers $n$ by (22) and

$$
g\left(2^{u}\right)=2^{u-1}-1 \text { if } u \geqq 1 ; g(m)=\sum_{m=q q^{\prime}}\left(2 \mid q^{\prime}\right) q .
$$

If $d=12$, the genus of $F_{12}$ consists of two classes, that of $F_{12}$ and that of

$$
F_{12}^{\prime \prime}=4 t_{0}^{2}+3\left(3 t_{1}^{2}+3 t_{2}^{2}+3 t_{3}^{2}-2 t_{2} t_{3}-2 t_{3} t_{1}-2 t_{1} t_{2}\right) \text {. }
$$

Since $F_{12}$ and $F_{12}^{\prime \prime}$ have the same number of unimodular automorphs, classical methods yields a formula not unlike that for $F_{5}$, for $F_{1}(n)+F_{2}(n)$, where $F_{1}(n)$ and $F_{2}(n)$ denote the number of representations of $n$ by $F_{12}$ and $F_{12}^{\prime \prime}$ respectively. If $n$ is a norm and positive, we can write

$$
\begin{aligned}
& n=s_{0}^{2}-12 s_{3}^{2}=2^{u} 3^{v} m, \text { where } u=0 \text { or } u \geqq 2, v \geqq 0, \text { and } \\
& m \equiv 1(\bmod 12) \text { if } u+v \text { is even, } m \equiv-1(\bmod 12) \text { if } u+v \text { is odd. }
\end{aligned}
$$

It can be shown for $n$ a positive norm that $F_{1}(n)+F_{2}(n)=2 g(n)$, where $g(n)$ is the factorable function defined for all positive integers $n$ (whether norms or not) by (22) and

$$
\begin{aligned}
& g\left(2^{u}\right)=\left|2^{u}-2\right|(u \geqq 0), g\left(3^{v}\right)=\left(3^{v}+1\right) / 2, \\
& g(m)=\sum_{m=q q^{\prime}}\left(2 \mid q^{\prime}\right) q .
\end{aligned}
$$

In $\$ 10$ we will prove

THEOREM 7. $F_{1}(n)=F_{2}(n)$ if $n$ is even, or if $n$ is divisible to an odd exponent by 3 or by any prime $p$ such that $p \equiv-1(\bmod 12)$.

By a familiar argument in elementary number theory, if $g(n)$ is a factorable function, and $g^{\prime}(n)$ is the factorable function defined by $g^{\prime}\left(p^{a}\right)=g\left(p^{a}\right)-g\left(p^{a-2}\right)$ if $a \geqq 2, g^{\prime}\left(p^{a}\right)=g\left(p^{a}\right)$ if $a=0$ or 1 , then $g(n)=\Sigma g^{\prime}\left(n / q^{2}\right)$, summed over the square factors $q^{2}$ of $n$. From this remark readily follows

THEOREM 8. If $D$ is a divisor of $n$, the number of solutions of

$$
n=t_{0}^{2}+d\left(t_{1}^{2}+t_{2}^{2}+t_{3}^{2}\right),\left(t_{0}, t_{1}, t_{2}, t_{3}, D\right)=h^{\prime},
$$


is $2 g_{D / h^{\prime}}\left(n / h^{\prime 2}\right)$ if $d=5$ or $8 ;$ but $g_{D / h^{\prime}}\left(n / h^{\prime 2}\right)$ if $d=12$ and $F_{1}\left(n / h^{\prime 2}\right)=F_{2}\left(n / h^{\prime 2}\right)$. Here $g_{k}(n)$ is the "simultaneously factorable" function defined for positive integers $n$, and factors $k$ of $n$ as follows. If $n=n_{1} n_{2}$ with $\left(n_{1}, n_{2}\right)=1$, we can write $k=k_{1} k_{2}$, where $k_{1}$ divides $n_{1}$ and $k_{2}$ divides $n_{2}$, and require that

$$
\begin{gathered}
g_{k}(n)=g_{k_{1}}\left(n_{1}\right) g_{k_{2}}\left(n_{2}\right), \\
\text { if } n=p^{a} \text { and } k=p^{b}(0 \leqq b \leqq a), \\
\text { then } g_{k}(n)=g\left(p^{a}\right) \text { if } a=0 \text { or } 1, \text { or } b=0, \\
g_{k}(n)=g\left(p^{a}\right)-g\left(p^{a-2}\right) \text { if } a \geqq 2 \text { and } b>0 .
\end{gathered}
$$

6. Discussion of $h$ and $l$ when $d=5$. To obtain $r_{4}(\sigma, 5)$ by (6), we must, for each solution $t_{0}, \cdots, t_{3}$ of (7) for which $b=\left(2 s_{0}+s_{1}-2 t_{0}\right) / 5$ is an integer, construct $r_{4}(l h)$ and evaluate the sum of the numbers $r_{4}(l h)$. Here $h=$ $\left(t_{1}, t_{2}, t_{3}, e\right)$, where $e=\left(s_{0},\left(s_{1}-b\right) / 2, b\right)$; and $l=2$ if the power of 2 in $b-s_{1}$ does not exceed that in $\left(s_{0}, b\right)$, otherwise $l=1$. Since $r_{4}(2 h)=r_{4}(h)$ when $h$ is even, the value of $l$ need only be considered when $h$ is odd.

Inserting the components of $e$, and noting by (7) that the g.c.d. of $s_{0}, s_{1}, t_{1}, t_{2}, t_{3}$ must divide $t_{0}$, we have

$$
\begin{aligned}
h & =\left(t_{1}, t_{2}, t_{3}, s_{0},\left(2 s_{0}+s_{1}-2 t_{0}\right) / 5,\left(s_{0}-2 s_{1}-t_{0}\right) / 5\right) \\
& =\left(t_{1}, t_{2}, t_{3}, s_{0}, s_{1},\left(s_{0}-2 s_{1}-t_{0}\right) / 5\right) \\
& =\left(t_{0}, t_{1}, t_{2}, t_{3}, s_{0}, s_{1},\left(s_{0}-2 s_{1}-t_{0}\right) / 5\right), \\
h & =\left(t_{0}, t_{1}, t_{2}, t_{3}, s_{0}, s_{1},\left(2 s_{0}+s_{1}-2 t_{0}\right) / 5\right) .
\end{aligned}
$$

We must determine whether $h=h^{\prime}$ or $h^{\prime} / 5$, where

$$
h^{\prime}=\left(t_{0}, t_{1}, t_{2}, t_{3}, s_{0}, s_{1}\right) \text {. }
$$

Evidently, $h=h^{\prime}$ if either $\left(t_{0}, t_{1}, t_{2}, t_{3}\right)$ is prime to 5 , or $v<2$ (in (20)), since in the last case $D=\left(s_{0}, s_{1}\right)$ is prime to 5 . Hence, if $5^{y}$ denotes the power of 5 in $\left(t_{0}, t_{1}, t_{2}, t_{3}\right)$, we can suppose $v \geqq 2, y>0$, that is $0<2 y \leqq v$.

If $y<v / 2$, then $5^{y+1} \mid t_{0}$. Notice from

$$
\left(2 s_{0}+s_{1}\right)^{2}-5 s_{1}^{2}=2^{2 u+2} 5^{v} m
$$

that if $v$ is even, $5^{v} \|\left(2 s_{0}+s_{1}\right)^{2}$ and $5^{v} \mid s_{1}^{2}$; and if $v$ is odd, $5^{v+1} \mid\left(2 s_{0}+s_{1}\right)^{2}$ and $5^{v-1} \| s_{1}^{2}$. Hence if $y<v / 2,5^{y+1} \mid\left(2 s_{0}+s_{1}-2 t_{0}\right)$, and so $h=h^{\prime}$.

But if $y=v / 2>0$, then $\left(2 s_{0}+s_{1}\right)^{2}-\left(2 t_{0}\right)^{2}$ is divisible by $5^{2 y+1}$, hence one of $2 s_{0}+s_{1}+2 t_{0}$ and $2 s_{0}+s_{1}-2 t_{0}$ is divisible by $5^{y+1}$, the other by $5^{y}$ (but not by $5^{y+1}$, since $5^{y+1}$ does not divide $t_{0}$ ). Hence

$$
h=h^{\prime} \text { for one sign of } t_{0}, h=h^{\prime} / 5 \text { for the other. }
$$

If $u=0, l=1$. For $s_{0}$ or $b$ is odd. 
If $u=1$, then $s_{0}, s_{1}, t_{0}, t_{1}, t_{2}, t_{3}$ must be even, and it is immaterial whether $l$ is 1 or 2 . If $u \geqq 2$ and $t_{0}, \cdots, t_{3}$ are odd, then $l=2$, since $s_{0} \equiv s_{1} \equiv 0, b \equiv 2$ $(\bmod 4)$.

7. The formula for $r_{4}(\sigma, 5)$. We can now reformulate (6) in terms of $h^{\prime}$ rather than $h$. If $h^{\prime}$ is any divisor of $D=\left(s_{0}, s_{1}\right)$, then the condition that $\left(t_{0}, t_{1}, t_{2}, t_{3}, s_{0}, s_{1}\right)=h^{\prime}$ means, on setting $t_{i}=h^{\prime} u_{i}$, that $u_{0}, \cdots, u_{3}$ are solutions of (31), with $n$ replaced by $n / h^{\prime 2}$ and $k=D / h^{\prime}$. Let $\zeta(q)$ denote the sum of the divisors of $q$. Now $r_{4}(l h)$ is the product of the numbers $\zeta\left(p^{r}\right)$ ( $p^{r}$ ranging over the prime-powers in $h$ ), times 8 or 24 according as $l h$ is odd or even. To compensate for the case of (36) we can use in place of $\zeta\left(5^{r}\right)$ (where $5^{r} \| h^{\prime}$ ) the factor $\left(\zeta\left(5^{r}\right)+\zeta\left(5^{r-1}\right)\right) / 2$. Thus:

$$
r_{4}(\sigma, 5)=8 \varepsilon \sum_{h^{\prime} \mid D} g_{D / h^{\prime}}\left(n / h^{\prime 2}\right) \psi_{n}\left(h^{\prime}\right),
$$

where $\varepsilon=1$ if $v=0, \varepsilon=2$ if $v>0$; and $\psi_{n}\left(h^{\prime}\right)$ is the factorable function defined as follows:

$$
\begin{aligned}
\psi_{n}\left(2^{r}\right) & =1 \text { if } r=0 ; \\
& =3 \text { if } r>0 ; \\
\psi_{n}\left(5^{r}\right) & =\left(\zeta\left(5^{r}\right)+\zeta\left(5^{r-1}\right)\right) / 2, \text { if } v(\geqq 2) \text { is even and } r=v / 2 ; \\
& =\zeta\left(5^{r}\right) \text { otherwise; } \\
\psi_{n}\left(p^{r}\right) & =\zeta\left(p^{r}\right), \text { for all odd primes different from } 5 .
\end{aligned}
$$

For any prime $p$, let $p^{a}$ denote the power of $p$ in $D=\left(s_{0}, s_{1}\right)$, and let $p^{2 a+b}$ denote the power of $p$ in $n=N \sigma$. Then by the multiplicative properties of $g_{k}(q)$ and $\psi_{n}\left(h^{\prime}\right), r_{4}(\sigma, 5) /(8 \varepsilon)$ is the product for all $p$ of

$$
c_{p}=\sum_{r=0}^{a} g_{p^{a-r}}\left(p^{2 a+b-2 r}\right) \psi_{n}\left(p^{r}\right)
$$

The evaluation of $c_{p}$ is straightforward, and gives the following results: If $p$ is an odd prime different from 5 , and $w=(5 \mid p)$,

$$
c_{p}=\frac{p^{a+b+1}-w^{b+1}}{p-w} \cdot \frac{p^{a+1}-1}{p-1} .
$$

If $p=5$, then $v=2 a+b$, and, in all cases,

$$
\varepsilon c_{5}=\left(5^{v+1}-1\right) / 4 \text {. }
$$

If $p=2$, then $a=u$ and $b=0$, and, in all cases,

$$
c_{2}=1 \text { if } u=0, c_{2}=2^{2 u+1}-5 \text { if } u>0 .
$$

8. Discussion of $h$ and $l$ when $d=8$. We use the notations in (25)-(26). Note that $b=\left(s_{0}-t_{0}\right) / 4$ is integral only by choice of sign of $t_{0}$ if $u=0$, and is always integral if $u>0$. Also, $e=\left(s_{0}, s_{3}, b\right)$, and $h=\left(t_{1}, t_{2}, t_{3}, e\right)$, hence 


$$
h=\left(t_{0}, t_{1}, t_{2}, t_{3}, s_{0}, s_{3},\left(s_{0}-t_{0}\right) / 4\right) .
$$

We choose $h^{\prime}=\left(t_{0}, t_{1}, t_{2}, t_{3}, s_{0}, s_{3}\right)$. To construct a formula similar to (37), we must try to define $\psi_{n}\left(h^{\prime}\right)$ in connection with $r_{4}(l h)$ in such a manner that it compensates for the cases where $h^{\prime}$ and $l h$ have different parities.

A curious situation appears when $u=2$. Then $t_{0} \equiv s_{0} \equiv 2(\bmod 4)$. If $m \equiv 1$ $(\bmod 8), s_{3}$ is even, $t_{1}, t_{2}, t_{3}$ are even: hence $h^{\prime}$ is double-odd, while $h$ is odd or double-odd according to the sign of $t_{0}$; but $l=1$ since $s_{2}$ contains no higher power of 2 than $s_{3}$. If $m \equiv-1(\bmod 8), s_{3}$ is odd, and $h=h^{\prime}$ (both odd); since $\left(s_{0}-t_{0}\right) / 4$ changes parity with the sign of $t_{0}, l$ alternates between 1 and 2 with that sign. In both cases the exponent of 2 in $h^{\prime}$ is unique for all solutions of (7). To keep $\psi_{n}\left(h^{\prime}\right)$ factorable we can define $\psi_{n}\left(2^{r}\right)=1(r \geqq 0)$ when $u=2$, and can compensate both for the present phenomenon and for the need to make $b$ integral, by defining

$$
\varepsilon=1 \text { if } n \text { is odd; } \varepsilon=4 \text { if } n \equiv 4(\bmod 8), \varepsilon=2 \text { if } n \equiv 0(\bmod 8) .
$$

We will prove for $u>2$ that $h^{\prime} \equiv l h(\bmod 2)$. First let $2^{3} \| N \sigma$. Then $s_{3}$ is odd, hence $h$ and $h^{\prime}$ are odd. Also $l=2$ leads to a contradiction: then $\left(s_{0}-t_{0}\right) / 4$ is even, hence since 4 divides $s_{0}$ and $t_{0}$,

$$
8\left(t_{1}^{2}+t_{2}^{2}+t_{3}^{2}\right) \equiv s_{0}^{2}-t_{0}^{2}-8 s_{3}^{2} \equiv-8(\bmod 64)
$$

Second, if $16 \mid N \sigma$, then either $s_{0} \equiv t_{0} \equiv 0$ or $4(\bmod 8)$, hence $h$ and $h^{\prime}$ are even; or $s_{0} \equiv t_{0}+4 \equiv 0$ or $4(\bmod 8)$, hence $\pm 2 \equiv s_{3}^{2}+t_{1}^{2}+t_{2}^{2}+t_{3}^{2}(\bmod 8)$, hence $h$ and $h^{\prime}$ are odd and $l=1$.

9. The formula for $r_{4}(\sigma, 8)$. In much the same way as in $\S 1$, we have

$$
r_{4}(\sigma, 8)=8 \varepsilon \sum_{h^{\prime} \mid D} g_{D / h^{\prime}}\left(n / h^{\prime 2}\right) \psi_{n}\left(h^{\prime}\right)=8 \varepsilon c_{2} \prod_{p \text { odd }} c_{p}
$$

where $D=\left(s_{0}, s_{3}\right), n=N \sigma, \varepsilon$ is defined in (44), $\psi_{n}$ is the factorable function such that $\psi_{n}\left(p^{r}\right)=\zeta\left(p^{r}\right)$ for odd primes $p, \psi_{n}\left(2^{r}\right)=1$ if $8 \nmid n$, and

$$
\psi_{n}\left(2^{r}\right)=1 \text { or } 3 \text { according as } r=0 \text { or } r>0 \text {, if } 8 \mid n \text {. }
$$

The evaluation of the factors $c_{p}$ is straightforward: $c_{p}$ is given by (40) with $w=(2 \mid p)$ if $p$ is any odd prime, and $\varepsilon c_{2}$ has the value

$$
1 \text { if } u=0 ; 4 \text { if } u=2 ; 6\left(2^{u-2}-1\right) \text { if } u>2 .
$$

10. Discussion of $h$ and $l$ when $d=12$. We use (28), $e=\left(s_{0}, s_{3}, j\right)$, where

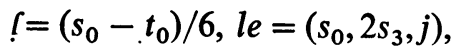

$$
h=\left(h^{\prime}, j\right) \text {, where } h^{\prime}=\left(t_{0}, \cdots, t_{3}, s_{0}, s_{3}\right) .
$$

The integrality of $j$ depends on the sign of $t_{0}$ if $(n, 3)=1$.

We prove for $n$ odd that $h=h^{\prime}$ and $l=1$. For, since $s_{0}$ is odd, $h^{\prime}$ and $h$ are odd, and $l=1$. If $(n, 3)=1, s_{0}, h^{\prime}$, and $h$ are prime to 3 , hence $h=h^{\prime}$. If $3 \mid n$, 
we assume (cf. Theorem 7) that $v$ is odd. Let $3^{b} \|\left(t_{0}, \cdots, t_{3}\right)$. Then $2 b+1 \leqq v$, $3^{b}\left|\left(s_{0}, s_{3}\right), 3^{b+1}\right| s_{0}, 3^{2 b+1}\left|t_{0}^{2}, 3^{b+1}\right| t_{0}, 3^{b} \mid j, h=h^{\prime}$.

Case $n$ even. We can set $s_{0}=2 s_{2}, t_{0}=2 t_{0}^{\prime \prime}$, and have $j=\left(s_{2}-t_{0}^{\prime \prime}\right) / 3$,

$$
2^{u-2} 3^{v} m=s_{2}^{2}-3 s_{3}^{2}=t_{0}^{\prime \prime 2}+3\left(t_{1}^{2}+t_{2}^{2}+t_{3}^{2}\right) \text {. }
$$

Denote by $2^{a}, 3^{b}$ the powers of 2 and 3 in $\left(t_{0}, t_{1}, t_{2}, t_{3}\right)$. If $2^{a-1} \| t_{0}^{\prime \prime}$, then $u=2 a$, $s_{2}=2^{a-1} 3^{b} s_{4}, s_{3}=2^{a-1} 3^{b} s_{5}$, and $s_{4}^{2}-3 s_{5}^{2}$ is odd. If $2^{a} \mid t_{0}^{\prime \prime}, u \geqq 2 a+2, s_{2}=2^{a} 3^{b} s_{4}$, $s_{3}=2^{a} 3^{b} s_{5}$. Also, $v \geqq 2 b$; and if $v=2 b,\left(s_{4}, 3\right)=1$.

We prove that $h$ and $h^{\prime}$ contain the same power of 2 . Indeed, if $s_{5}$ is odd, the power of 2 in $s_{3}$ divides both $s_{2}$ and $t_{0}^{\prime \prime}$. If $s_{5}$ is even, then if $2^{a}\left|t_{0}^{\prime \prime}, 2^{a}\right| j$; and if $2^{a-1} \| t_{0}^{\prime \prime}, 3 j=2^{a-1}($ odd $)-2^{a-1}($ odd $) \equiv 0\left(\bmod 2^{a}\right)$.

We prove next that $h$ is odd and $l=2$ if and only if

$$
s_{2} \text { even, } s_{3} \text { odd, }\left(t_{0}, t_{1}, t_{2}, t_{3}\right) \text { odd. }
$$

For if $h$ is odd, $s_{3}$ or $\left(t_{1}, t_{2}, t_{3}\right)$ is odd. By (49), $s_{3}$ even and $j$ even imply that $4 \mid\left(t_{1}, t_{2}, t_{3}\right)$. Hence if $h$ is odd and $s_{3}$ is even, $j$ is odd and $l=1$. Thus, if $h$ is odd and $l=2$, then $j$ must be even; also, $s_{2}$ must be even, since otherwise (49) implies $t_{1}^{2}+t_{2}^{2}+t_{3}^{2} \equiv-1(\bmod 8)$. Finally, notice that if $s_{2}$ is even and $s_{3}$ is odd, then $j$ is even (hence $l=2$ ) if and only if $\left(t_{1}, t_{2}, t_{3}\right)$ is odd.

We prove finally that $h=h^{\prime}$ except that when $v$ is even and positive, and $3^{v / 2} \|\left(t_{0}, t_{1}, t_{2}, t_{3}\right)$, then $h=h^{\prime}$ for one sign of $t_{0}, h=h^{\prime} / 3$ for the other. First, if $v=0, h^{\prime}$ is prime to 3 , hence $h=h^{\prime}$. If $v>0$, (49) can be divided by the powers of 2 and 3 dividing $\left(t_{0}^{\prime \prime}, t_{1}, t_{2}, t_{3}\right)$, and gives

$$
s_{4}^{2}-t_{0}^{\prime 2}=3 \text { times an integer. }
$$

If here $v>2 b$, then $3 \mid\left(s_{4}, t_{0}^{\prime}\right)$ and $h=h^{\prime}$. But if $v=2 b,\left(s_{4}, 3\right)=1$, hence only one of $s_{4}+t_{0}^{\prime}$ and $s_{4}-t_{0}^{\prime}$ is divisible by 3 , and $h=h^{\prime}$ or $h^{\prime} / 3$ correspondingly.

11. Proof of Theorem 7; evaluation of $r_{4}(\sigma, 12)$ in certain cases. To compensate for the final result of $\S 10$ is easy: simply define

$$
\begin{aligned}
\psi_{n}\left(3^{b}\right) & =\zeta\left(3^{b}\right) & & \text { if } 2 b<v \text { or } b=0, \\
& =\left(\zeta\left(3^{b}\right)+\zeta\left(3^{b-1}\right)\right) / 2 & & \text { if } 2 b=v>0 .
\end{aligned}
$$

If $p>3$, we let $\psi_{n}\left(p^{b}\right)=\zeta\left(p^{b}\right)$. And if $p=2$, we let $\psi_{n}\left(2^{b}\right)=1$ if $b=0, \psi_{n}\left(2^{b}\right)=3$ if $b>0$. This does not represent the needs of the case $u=2$ (cf. (50)), but this case will be handled separately below.

We proceed with the proof of Theorem 7, and refer to the paragraph of (27).

LEMMA 1. If $n=4 q$, $q$ integral, then $F_{1}(n)=F_{2}(n)$.

Proof. $4 q=t_{0}^{2}+12\left(t_{1}^{2}+t_{2}^{2}+t_{3}^{2}\right)$ reduces to $q=t_{0}^{\prime \prime 2}+3\left(t_{1}^{2}+t_{2}^{2}+t_{3}^{2}\right)$. In (27), $3 t_{1}^{2}+\cdots-2 t_{1} t_{2}-\cdots=\left(t_{2}+t_{3}-t_{1}\right)^{2}+\cdots+\left(t_{1}+t_{2}-t_{3}\right)^{2}$. Now 4 divides a sum of three squares only if they are even. Finally, 


$$
t_{2}+t_{3}-t_{1}=2 x_{1}, \cdots, t_{1}+t_{2}-t_{3}=2 x_{3},
$$

gives $t_{1}=x_{2}+x_{3}, \cdots, t_{3}=x_{1}+x_{2}$, and replaces $\frac{1}{4} F_{12}^{\prime \prime}$ by $t_{0}^{2}+3\left(x_{1}^{2}+x_{2}^{2}+x_{3}^{2}\right)$.

Besides multiples of $4, F_{12}$ and $F_{12}^{\prime \prime}$ represent only odd numbers of the form $4 q+1$. Consider now the equation

$$
4 q+1=t_{0}^{\prime 2}+3\left(t_{1}^{2}+t_{2}^{2}+t_{3}^{2}\right)
$$

LEMMA 2. There is a one-to-one association between the solutions of (53) with $t_{0}^{\prime \prime}$ respectively odd and even, and the representations of $4 q+1$ by $F_{12}$ and $F_{12}^{\prime \prime}$.

Proof. If $t_{0}^{\prime \prime}$ is odd, $t_{1} t_{2}, t_{3}$ are even. If $t_{0}^{\prime \prime}$ is even, then $t_{1}, t_{2}, t_{3}$ are odd and integers $u_{j}$ can be chosen so that $u_{2}+u_{3}-u_{1}=t_{1}, \cdots, u_{1}+u_{2}-u_{3}=t_{3}$.

Some comments will now be made on the cases $N \sigma$ odd or quadruple-odd.

In formulae (54)-(57), $\sigma$ will designate a number $s_{0}+2 s_{3} \omega$ with $s_{0}$ odd; $\sigma^{\prime}$, one with $s_{0}$ divisible by 4 and $s_{3}$ odd; $\sigma^{\prime \prime}$, one with $s_{0}$ double-odd, $s_{3}$ even. The odd part of the divisor $\left(s_{0}, s_{3}\right)$ will be assumed to be the same for all three. If we wish, we can take $\sigma^{\prime \prime}=2 \sigma, \sigma^{\prime}=2 \theta \sigma$, where $\theta$ is the unit $2+3^{1 / 2} ; N \sigma=k, N \sigma^{\prime}$ $=N \sigma^{\prime \prime}=4 k, k=4 q+1$.

Let $D=\left(s_{0}, s_{3}\right), k=4 q+1$, and for any divisor $j$ of $D$, let $F_{i, j}\left(k / j^{2}\right)$ denote the number of solutions of $(53)$ with $t_{0}^{\prime \prime} \equiv i(\bmod 2),\left(2 t_{0}^{\prime \prime}, t_{1}, t_{2}, t_{3}, D\right)=j$.

Since $N \sigma=k$ is odd, the factors of $D$ are odd, $l=1$, and hence

$$
r_{4}(\sigma, 12)=4 \varepsilon \sum_{j \mid D} F_{1, j}\left(k / j^{2}\right) \cdot \psi_{k}(j)
$$

with $\varepsilon=1$ or 2 according as $k$ is or is not prime to 3 .

Consider now $\sigma^{\prime}$. In deriving (50) we noticed that $h^{\prime}$ is odd, and that $l=2$ if $t_{0}^{\prime \prime}$ is even (corresponding, we now recognize, to a representation of $k$ by $F_{12}^{\prime \prime}$ ), and $l=1$ if $t_{0}^{\prime \prime}$ is odd (corresponding to a representation of $k$ by $F_{12}^{\prime}$ ). Accordingly,

$$
r_{4}\left(\sigma^{\prime}, 12\right)=4 \varepsilon \sum_{j \mid D}\left\{3 F_{1, j}\left(k / j^{2}\right)+F_{0, j}\left(k / j^{2}\right)\right\} \cdot \psi_{n}(j)
$$

In the case of $\sigma^{\prime \prime}, h^{\prime}$ odd means that $\left(t_{1}, t_{2}, t_{3}\right)$ is odd, corresponding to a representation of $k$ by $F_{12}^{\prime \prime}$. And $h^{\prime}$ even means $t_{1}, t_{2}, t_{3}$ even, corresponding to a representation of $k$ by $F_{12}$. Hence:

$$
r_{4}\left(\sigma^{\prime \prime}, 12\right)=4 \varepsilon \sum_{j \mid D}\left\{F_{1, j}\left(k / j^{2}\right)+3 F_{0, j}\left(k / j^{2}\right)\right\} \psi_{n}(j)
$$

Since $k$ is odd, a simplifying expression for $F_{i, j}\left(k / j^{2}\right)$ is not in general available. However, since as noted following $(28), F_{2}(n)+F_{1}(n)=2 g(n)$, we have $F_{0, j}\left(k / j^{2}\right)+F_{1, j}\left(k / j^{2}\right)=2 g_{D / j}\left(k / j^{2}\right)$, and hence 


$$
\begin{aligned}
\left\{r_{4}\left(\sigma^{\prime}, 12\right)+2 r_{4}(\sigma, 12)\right\} / 3 & =r_{4}\left(\sigma^{\prime \prime}, 12\right)-2 r_{4}(\sigma, 12) \\
& =4 \varepsilon \sum_{j \mid D !}\left\{F_{0, j}\left(k / j^{2}\right)+F_{1, j}\left(k / j^{2}\right)\right\} \psi_{k}(j) \\
& =8 \varepsilon \sum_{j \mid D} g_{D / j}\left(k / j^{2}\right) \psi_{k}(j) \\
& =8 \varepsilon \prod_{p \text { odd }} c_{p},
\end{aligned}
$$

where $c_{p}$ is given by (40) with $w=(3 \mid p)$ if $p>3$, and $c_{3}=\left(3^{v+1}-1\right) / 2$ if $3^{v} \| k$. From (57) also follows that $r_{4}\left(\sigma^{\prime}, 12\right)+r_{4}\left(\sigma^{\prime \prime}, 12\right)=32 \varepsilon \prod c_{p}$.

Let $G_{i}(n)$ denote the number of solutions of

$$
n=3 x_{0}^{2}+x_{1}^{2}+x_{2}^{2}+x_{3}^{2}, x_{0} \equiv i(\bmod 2),
$$

$(i=1,2)$. Clearly $F_{i}(3 n)=G_{i}(n)$ for any integer $n$.

LEMMA 3. If $n \equiv 5(\bmod 6), G_{2}(n)=G_{1}(n)$.

Proof. In (58), two of $x_{1}, x_{2}, x_{3}$ are prime to 3 , hence exactly half the solutions satisfy $3 \mid x_{1}+x_{2}+x_{3}$. The self-inverse substitution

$$
y_{0}=\left(x_{1}+x_{2}+x_{3}\right) / 3, y_{1}=x_{0}+\left(2 x_{1}-x_{2}-x_{3}\right) / 3, \cdots, \cdots,
$$

under which $3 x_{0}^{2}+\sum x_{j}^{2}=3 y_{0}^{2}+\sum y_{j}^{2}$, carries $x_{0}$ into $y_{0}$ of the opposite parity.

LEMMA 4. If $n$ is odd, and $\phi(n)$ denotes the number of solutions of

$$
n=y_{0}^{2}+y_{1}^{2}+2 y_{2}^{2}+2 y_{2} y_{3}+2 y_{3}^{2} \text {, }
$$

then

$$
G_{i}(3 n)+3 F_{i}(n)=2 \phi(n) \quad(i=1,2)
$$

hence

$$
F_{2}(9 n)+3 F_{2}(n)=F_{1}(9 n)+3 F_{1}(n) .
$$

Proof. In $3 n=3 x_{0}^{2}+x_{1}^{2}+x_{2}^{2}+x_{3}^{2}$, either $x_{3} \equiv \pm x_{1} \equiv \pm x_{2}(\bmod 3)$ for exactly one of the four combinations of signs, or 3 divides $\left(x_{1}, x_{2}, x_{3}\right)$. The discussion for $x_{1} \equiv-x_{2} \equiv x_{3}$ will be typical. Then $x_{0}=u_{0}, x_{1}=u_{1}, x_{2}=3 u_{2}-u_{1}$, $x_{3}=3 u_{3}+u_{1}, 3 n=3 u_{0}^{2}+3 u_{1}^{2}+9 u_{2}^{2}-6 u_{1} u_{2}+9 u_{3}^{2}+6 u_{1} u_{3}$, or

$$
n=u_{0}^{2}+\left(u_{1}-u_{2}-u_{3}\right)^{2}+2 u_{2}^{2}+2 u_{2} u_{3}+2 u_{3}^{2} \text {. }
$$

Thus $G_{i}(3 n)=F_{i}(n)+4\left\{\phi_{i}(n)-F_{i}(n)\right\}(i=1,2)$, where $\phi_{i}(n)$ denotes the number of solutions of $(60)$ with $y_{0} \equiv i(\bmod 2)$. But since $n$ is odd, $y_{0}$ is odd or even equally often in (60), $2 \phi_{i}(n)=\phi(n)$.

CoROllary. $\quad F_{2}(k)=F_{1}(k)$ if $k=3^{h} m(m \equiv 5 \bmod 6, h>0)$, hence if $k$ is a norm divisible by 3 to an odd exponent. 
Proof. If $n \equiv 2(\bmod 3)$, Lemma 3 shows that $F_{2}(3 n)=F_{1}(3 n)$, and hence by $(61), F_{2}\left(3^{2 h+1} n\right)=F_{1}\left(3^{2 h+1} n\right)(n \equiv 5 \bmod 6, h \geqq 0)$. Also, by (61), since $F_{2}(n)=F_{1}(n)=0$ if $n \equiv 2(\bmod 3)$, also $F_{2}\left(3^{2 h+2} n\right)=F_{1}\left(3^{2 h+2} n\right)$ if $n \equiv 5$ $(\bmod 6)$ and $h \geqq 0$.

LEMMA 5. Let $p$ denote an odd prime such that $(3 \mid p)=1$. There are $p-(-1 \mid p)$ matrices $R$ of determinant $v(\bmod p)$ which satisfy $R^{\prime} E R \equiv-3 I$ $(\bmod p)$, where

$$
E=\left(\begin{array}{ll}
1 & 0 \\
0 & 3
\end{array}\right), \quad I=\left(\begin{array}{ll}
1 & 0 \\
0 & 1
\end{array}\right), \text { and } R \text { can be designated as }\left(\begin{array}{ll}
a & b \\
c & d
\end{array}\right),
$$

and where $v$ denotes a fixed solution of $v^{2} \equiv 3(\bmod p)$. Consider the $p-(-1 \mid p)$ formulae

$$
x_{0} \equiv a x_{2}+b x_{3}, x_{1} \equiv c x_{2}+d x_{3}(\bmod p) \text {. }
$$

Trivially, if $x_{2}=x_{3}=0,(63)$ gives the null solution $x_{0}=x_{1}=x_{2}=x_{3}=0$ of

$$
x_{0}^{2}+3 x_{1}^{2}+3 x_{2}^{2}+3 x_{3}^{2} \equiv 0(\bmod p) \text {. }
$$

As $x_{2}, x_{3}$ range over the $p^{2}-1$ pairs not 0,0 , the $p-(-1 \mid p)$ formulae (63) give distinct solutions of (64). Every solution of (64) is so given if $p \equiv 3(\bmod 4)$. But if $p \equiv 1(\bmod 4)$, there are exactly $2\left(p^{2}-1\right)$ additional non-null solutions of (64) which satisfy

$$
x_{0} \equiv u v x_{1}, x_{2} \equiv u x_{3}(\bmod p) \text {, where } u^{2} \equiv-1(\bmod p) .
$$

Proof. First notice that (63) and (63') do provide solutions of (64). For example, $R^{\prime} E R \equiv-3 I(\bmod p)$ expands into

$$
a^{2}+3 c^{2} \equiv-3, b^{2}+3 d^{2} \equiv-3, a b+3 c d \equiv 0(\bmod p) .
$$

Notice also that $|R|^{2} \equiv 3(\bmod p)$, so that $|R| \equiv v$ or $-v(\bmod p)$. Also, if $|R|=v$, then multiplying the third member of (65) by $a$ or $b$, and using all of (65), we see that

$$
a \equiv-d v, b \equiv c v(\bmod p) .
$$

Now it is well known that $(64)$ has exactly $1+(p+1)\left(p^{2}-1\right)$ solutions. The lemma then asserts that if $x$ is a non-null column vector $\left\{x_{2}, x_{3}\right\}$, and $R_{1}, R_{2}$ are distinct solutions of $R^{\prime} E R \equiv-3 I$ with the same determinant $v$, then $R_{1} \mathfrak{x}$ and $R_{2} \mathfrak{x}$ are distinct vectors. This follows from the fact that $\left|R_{1}-R_{2}\right| \neq 0$. To prove the last fact, notice first that $R^{\prime} E R \equiv-3 I$ has at least one solution (solve $b^{2}$ $+3 d^{2} \equiv-3$ and use $\left(65^{\prime}\right)$ ), and that if $R$ is one solution then every solution is given by $R V$, where $V$ ranges over the solutions of $V^{2} V \equiv I(\bmod p)$. Now the last congruence has exactly the $2\{p-(-1 \mid p)\}$ solutions 


$$
\left(\begin{array}{rr}
y & z \\
-z & y
\end{array}\right) \text { and }\left(\begin{array}{rr}
y & z \\
z & -y
\end{array}\right) \text {, where } y^{2}+z^{2} \equiv 1
$$

For $\left(66_{1}\right)$ evidently $|V-I|=y^{2}-2 y+1+z^{2}=2-2 y \neq 0$ unless $V=I$. Hence also $|R V-R| \neq 0$ unless $V=I$. (On the other hand it is seen using the same machinery that if the determinants of $R_{1}$ and $R_{2}$ are different, then $R_{1}-R_{2}$ is always singular, and that therefore no new solutions are obtained by using $-v$ in place of $v$ !)

All solutions of $(64)$ are thus accounted for if $p \equiv 3(\bmod 4)$. But if $p \equiv 1$ $(\bmod 4)$, we are short $2\left(p^{2}-1\right)$ solutions in using $(63)$. These are provided by the $4(p-1)$ solutions satisfying $\left(63^{\prime}\right)$ with exactly one of the pairs $x_{0}, x_{1}$ and $x_{2}, x_{3}$ null; and the $2(p-1)^{2}$ solutions satisfying $\left(63^{\prime}\right)$ with neither pair null. There is no overlapping with the solutions satisfying (63), since (63) and (63') together imply that $x_{0}=x_{1}=x_{2}=x_{3}=0$. The lemma follows.

Consider now the equation

$$
p n=x_{0}^{2}+3\left(x_{1}^{2}+x_{2}^{2}+x_{3}^{2}\right) .
$$

We assume (63), the treatment of $\left(63^{\prime}\right)$ being similar. We can thus set

$$
x_{0}=p y_{0}+a y_{2}+b y_{3}, x_{1}=p y_{1}+c y_{2}+d y_{3}, x_{2}=y_{2}, x_{3}=y_{3} \text {, }
$$

and so have $p-(-1 \mid p)$ substitutions each of determinant $p^{2}$. For each of these the resulting form in $y_{0}, \cdots, y_{3}$ may be designated as $p g^{*}$. Thus $g^{*}$ is integral, has determinant $3^{3}\left(p^{2}\right)^{2} / p^{4}=3^{3}$, and has first coefficient $p$.

Now there are two genera of determinant $3^{3}$ which have the same ordinal structure as regards the primes 2 and 3 as $f=x_{0}^{2}+3 x_{1}^{2}+3 x_{2}^{2}+3 x_{3}^{2}$. One is that of $f$, and the other is that of

$$
f^{\prime}=2 z_{0}^{2}+2 z_{0} z_{1}+2 z_{1}^{2}+3 z_{2}^{2}+3 z_{3}^{2} .
$$

Clearly, $g^{*}$ belongs to the genus of $f$ or $f^{\prime}$ according as $p \equiv 1$ or $2(\bmod 3)$ : in other words, since $(3 \mid p)=1$, according as $p \equiv 1$ or $-1(\bmod 12)$. Both genera are known to consist of one class.

Consider the case $p=12 q-1$. Then there exists a linear transformation of integral matrix $T=\left(t_{i j}\right)(i, j=0, \cdots, 3)$ and determinant $p^{2}$ which replaces $f$ by $p f^{\prime}$. Hence

$$
2 p=t_{00}^{2}+3 t_{10}^{2}+3 t_{20}^{2}+3 t_{30}^{2} \text {. }
$$

If $t_{00}$ could be odd, $\left(2 p-t_{00}^{2}\right) / 3$ would be congruent to $7(\bmod 8)$ and could not be a sum of three squares. Hence $t_{00}$ and, likewise, $t_{01}$ are even. Both $t_{02}$ and $t_{03}$ cannot be even since $|T|$ is odd; also, both cannot be odd, since

$$
3 p=t_{02}^{2}+3\left(t_{12}^{2}+t_{22}^{2}+t_{32}^{2}\right), 3 p=t_{03}^{2}+3\left(t_{13}^{2}+t_{23}^{2}+t_{33}^{2}\right),
$$

then requires that $t_{12}, t_{22}, t_{32}, t_{13}, t_{23}, t_{33}$ are even, and this contradicts 


$$
0=t_{02} t_{03}+3\left(t_{12} t_{13}+t_{22} t_{23}+t_{32} t_{33}\right) \text {. }
$$

Hence $t_{02} \neq t_{03}(\bmod 2)$.

It follows that in $x_{0}=t_{00} z_{0}+t_{01} z_{1}+t_{02} z_{2}+t_{03} z_{3}, x_{0}$ is congruent (mod 2) to a definite one of $z_{2}$ and $z_{3}$. Hence, the number of representations of an odd number by $f^{\prime}$ with $z_{2}$ odd being half the total number $f^{\prime}(n)$, we have, if $n$ is odd and $p \equiv-1(\bmod 12)$,

$$
F_{i}(p n)=F_{i}(n / p)+(p+1)\left\{\frac{1}{2} f^{\prime}(n)-F_{i}(n / p)\right\}, \quad(i=1,2),
$$

or

$$
F_{i}(p n)+p F_{i}(n / p)=\frac{1}{2}(p+1) \cdot f^{\prime}(n), \quad(i=1,2) .
$$

Corollary 1. If $(n, p)=1$ and $p \equiv-1(\bmod 12), F_{1}(p n)=F_{2}(p n)$. Also, $F_{1}\left(p^{2 h+1} n\right)=F_{2}\left(p^{2 h+1} n\right)$.

This completes the proof of Theorem 7.

CoROllary 2. If $N \sigma$ is odd and is divisible by 3 or by some prime $p=12 q-1$ to an odd exponent, then $r_{4}(\sigma, 12)$ is given by the same final expression $8 \varepsilon \prod c_{p}$ as in (57).

Among other deductions that can be easily made from (70), along with the formula for $F_{1}(n)+F_{2}(n)$ is the value of $F_{1}\left(p^{2 h}\right)$. In particular,

$$
F_{1}\left(p^{2}\right)=\left(p^{2}+1\right) / 2 \text {. }
$$

Consider next the case where $2^{u} \| N \sigma=s_{0}^{2}-12 s_{3}^{2}, u(\geqq 3)$ odd. Then

$$
s_{0}=2^{(u-1) / 2} s_{2}, s_{3}=2^{(u-3) / 2} s_{5},
$$

$s_{2}^{2}-3 s_{5}^{2} \equiv 2(\bmod 4)$, hence $s_{2}$ and $s_{5}$ are odd. Hence $h^{\prime}$ can be divisible at most by $2^{(u-3) / 2}$. Accordingly,

where

$$
\begin{aligned}
r_{4}(\sigma, d) & =4 \varepsilon \sum_{h^{\prime} \mid D} g_{D / h^{\prime}}\left(n / h^{\prime 2}\right) \psi_{n}\left(h^{\prime}\right) \\
& =4 \varepsilon c_{2} \prod c_{p},
\end{aligned}
$$

$$
\begin{aligned}
c_{2} & =\sum_{i=0}^{(u-3) / 2} g_{2}^{(u-3) / 2-i}\left(2^{:-2 i}\right) \cdot \psi_{n}\left(2^{i}\right) \\
& =6\left(2^{u-2}-1\right),(u \text { odd }, u \geqq 3) .
\end{aligned}
$$

Finally, let $2^{u} \| s_{0}^{2}-12 s_{3}^{2}, u$ even, $u \geqq 4$. Write $D^{\prime \prime}=\left(s_{2}, s_{3}\right)$, where $s_{2}=s_{0} / 2$. Then $2^{(u / 2)-1} \| D^{\prime \prime}$, and if we define $h^{\prime \prime}=\left(t_{0}, \cdots, t_{3}, s_{2}, s_{3}\right)$, evidently $h^{\prime \prime}$ and $h^{\prime}$ are alike even or odd. Hence 


$$
\begin{aligned}
r_{4}(\sigma, d) & =4 \varepsilon \sum_{h^{\prime \prime} \mid D^{\prime \prime}} g_{D^{\prime \prime} / h^{\prime \prime}}\left(n / h^{\prime 2}\right) \cdot \psi_{n}\left(h^{\prime \prime}\right) \\
& =4 \varepsilon c_{2} \prod c_{p},
\end{aligned}
$$

where $c_{p}$ is of course the same as before, and $c_{2}$ is found to have the same final formula as in (72), with $u$ even, $u \geqq 4$.

\section{REFERENCES}

1. H. Cohn, A numerical study of the representation of a totally positive integer as a sum of quadratic integral squares, Numerische Math. 1 (1959), 121-134.

2. - Decomposition into four integral squares in the fields of $2^{1 / 2}$ and $3^{1 / 2}$, Amer. J. Math82 (1960), 301-322.

3. - Cusp forms arising from Hilbert's modular functions for the field of $3^{1 / 2}$, Amer. J. Math. 84 (1962), 283-305.

4. L. E. Dickson, History of the theory of numbers, Vol. III, Carnegie Institution, Washington, D. C., 1923, pp. 228-229.

4a. J. Dzewas, Quadratsummen in reell-quadratischen Zahlkörpern, Math. Nachr. 21 (1960), 233-284.

5. F. Götzky, Über eine zahlentheoretische Anwendung von Modulfunktionen zweier Verändlichen, Math. Ann. 100 (1928), 411-437.

6. L. J. Mordell, On the representation of a binary quadratic form as a sum of squares of linear forms, Math. Z. 35 (1932), 1-15.

7. I. Niven, Integers of quadratic fields as the sum of squares, Trans. Amer. Math. Soc. 48 (1940), 405-417.

8. G. Pall, The structure of the number of representations function in a binary quadratic form, Trans. Amer. Math. Soc. 35 (1933), 491-509.

9. - On the arithmetic of quaternions, Trans. Amer. Math. Soc. 47 (1940), 487-500.

10. —_, Representation by quadratic forms, Canad. J. Math. 1 (1949), 344-364.

11. - Sums of two squares in a quadratic field, Duke Math. J. 18 (1951), 399-409.

12. G. Pall and O. Taussky, Application of quaternions to the representation of a binary quadratic form as a sum of four squares, Proc. Roy. Irish Acad. 58A (1957), 23-28.

13. C. L. Siegel, Lectures on the analytical theory of quadratic forms, Mimeographed notes, Princeton, 1935.

UNIVERSTTY OF ARIZONA,

TUCSON, ARIZONA

ILLINOIS INSTITUTE OF TECHNOLOGY,

Chicago, Illinois 